\title{
Arqueología y TIC. Hacia una enseñanza/aprendizaje transversal en Secundaria. Propuesta de Innovación Educativa del Grupo de Investigación en Arqueología de la Universidad de Murcia (España)
}

\author{
Alicia FERNÁNDEZ DÍAZ \\ Universidad de Murcia \\ aliciafd@um.es \\ Jaime VizCAÍNO SÁNCHEZ \\ Universidad de Murcia \\ javisa@um.es \\ María Milagrosa Ros SALA \\ Universidad de Murcia \\ milaros@um.es \\ Sebastián F. Ramallo AsENSiO \\ Universidad de Murcia \\ sfra@um.es
}

\section{Resumen:}

Este trabajo presenta una propuesta de innovación educativa en la didáctica de la Arqueología. Creemos que las aulas preuniversitarias son el marco idóneo para la puesta en marcha y desarrollo de un nuevo modelo pedagógico que aúne Arqueología y TIC. Nuestro objetivo es la mejora del proceso de enseñanza/aprendizaje, favoreciendo la adquisición de la competencia digital.

Palabras Clave: Arqueología; TIC; enseñanza secundaria; enseñanza/aprendizaje; competencia digital

Archeology and ICT. Forward a transverse teaching/learning in Spanish secondary education. Educational innovation proposal of the Archeology Research Team of the University of Murcia (Spain)

\begin{abstract}
:
This work presents an educational innovation proposal to archaeological education. We think the secondary classrooms are the suitable frame for training and development of a new pedagogical model which unites Archeology and ICT. Our aim is the improvement of the teaching and learning process, to achieving the Digital Competence.
\end{abstract}

Key Words: Archeology; ICT; secondary education; teaching/learning; digital competence.

\section{Referencia normalizada:}

Fernández Díaz, A., Vizcaíno Sánchez, J., Ros Sala, M. M. y Ramallo Asensio, S. F. (2014): Arqueología y TIC. Hacia una enseñanza/aprendizaje transversal en secundaria. Propuesta de innovación educativa del grupo de investigación en arqueología de la Universidad de Murcia (España). Historia y Comunicación Social. Vol. 19. Núm. Especial Marzo. Págs. 425-438. 
Sumario: 1. Introducción. 2. Metodología. 3. Directrices didácticas. 4. Conclusiones

\section{Introducción}

La asignatura Historia, una de las que concita mayor peso didáctico entre las ciencias sociales en los curricula de Educación Secundaria, se contempla hoy día más dinámica que hace unas pocas décadas, al albur del espectacular desarrollo que toda una serie de disciplinas que la auxilian han vivido en estos años. De entre estas últimas, la Arqueología ha tenido una amplia repercusión social como descubridora, interpretadora a la vez que constructora $\mathrm{y}$, finalmente, transmisora de unos bienes patrimoniales no solo de naturaleza material sino, lo que es realmente importante, de carácter inmaterial identitario, a los que diferentes agentes sociales han elevado a la categoría de bienes de "consumo" sin ir más allá de la propia imagen, la leyenda, la tradición,...; y todo ello en una sociedad como la del s. XXI que, si es algo, es la Sociedad del Conocimiento gracias a los avances en investigación y a la repercusión de los mecanismos de información-difusión rápida, instantánea, socializada,..; es decir, progreso investigador, inducido y difundido globalmente gracias al constante desarrollo de las TIC, probablemente una de las armas "sociales" más poderosas con las que hoy día contamos en cualquier ámbito del desarrollo social.

De estas mismas ventajas se ha nutrido de forma excepcional la disciplina arqueológica, de manera que ha dotado a todos los campos de la Historia de un útil instrumento de vertebración e imbricación con otras materias, no solo de las ciencias naturales en cuyo seno surgió a fines del s. XVIII, sino, también y sobre todo, de las ciencias puras; en este sentido, siempre ha parecido "coherente" que las inscripciones y/o fuentes literarias latinas o griegas, o el conocimiento filosófico en su origen o el teatro y la oratoria, la geografia, etc., sean descifradas, interpretadas, desde un conocimiento adecuado de las lenguas latina y griega, al igual que el arte debía ser el acompañamiento ineludible de la expresión visual y sensorial de las sociedades en el tiempo. Pero hay algo más, siempre ha de haberlo, pero ahora, tras los cambios que ha sufrido la disciplina arqueológica -como muchas otras, por supuesto- al amparo de los avances en otras disciplinas, ese más ha tomado carta de naturaleza convirtiéndola en multidisciplinar e interdisciplinar y no solo como "auxiliada" por otras ciencias sino, también, como promotora de avances en estas circunstancias que la hace cada vez más actual y presente en la sociedad en general y en los medios de comunicación en particular.

Todo ello le confiere, dentro de la asignatura Historia, un carácter de transversalidad que no solo compete a la Enseñanza Superior sino que concierne e importa al ámbito de aprendizaje cruzado y cooperativo de la Enseñanza Secundaria, como iniciación y estímulo a adquirir mayores competencias y unos márgenes de profesionalidad más diversos. Porqué no aunar, pues, transversalidad e interactividad con coordinación del aprendizaje, interdisciplinariedad con cooperación efectiva entre la Historia y otras asignaturas, y reseñar, entre los distintos campos de sus aplicaciones, 
las que induce la Arqueología científica como ámbito más conocido por la comunidad educativa por su imbricación en la valorización del Patrimonio natural, histórico, científico y artístico, o su necesaria relación con los orígenes de la humanidad, o las implicaciones actualistas de los cambios climáticos en diferentes cambios culturales y revueltas sociales que miran, en última estancia, a problemas orbitales y cosmogénicos muy de actualidad con la preocupación mundial por las circunstancias de la aceleración del cambio climático; es más, porqué no utilizar ciertos modelos de aprendizaje colaborativo en la aplicación de ciencias tanto sociales, como naturales o como experimentales y puras, cuando este tipo de enseñanzas están ampliamente relacionadas en la Arqueología.

Y es que el educador ha de tener muy presente que hablar de Arqueología es hablar de isótopos del carbono, o del plomo, o de ADN, o de fitolitos, o de polen, o de radiometría del C14, o de dietas deducidas por el C13, o de antropología física, o de sismicidad, o de las propiedades del uranio y el torio, o de estadística matemática, o de modelos matemáticos, o micromorfología, o de fosfatos en suelos, o de zoología, o de botánica, o del estudio de climas pasados para entender los futuros, o de música, o de bellas artes, o de ciencias del deporte, o de........

La Arqueología, en suma, ha acercado las ciencias experimentales a la Historia y, a través de su plasmación en el patrimonio material e inmaterial, ha acercado ambas a esa sociedad que, al fin y a la postre, es impulsora de las primeras y hacedora de la segunda. Negar la posibilidad de hacer más comprensibles y cercanas, a la par que amenas por la curiosidad que el aprendizaje induce en el discente -que también la enseñanza en el docente-, de algunas disciplinas/asignaturas que en la actualidad son tenidas como frías o áridas, por su carácter fundamentalmente objetivo, como Física, Química, Matemáticas,..., es impedir ir más allá al alumno y, en definitiva, a la sociedad futura; más cuando en este específico aprendizaje interdisciplinar, cooperativo y colaborativo, las TIC tienen un papel fundamental por la facilidad que imprimen a la enseñanza aprendizaje. Pero para ello los docentes han de verlo claro y querer llevarlo a la práctica (vid. infra los resultados de la encuesta realizada en este trabajo), por lo que una primera acción ha de ser con estos educadores que, a buen seguro, con las evidencias adecuadas, se sumarían a este modelo de aprendizaje interdisciplinar que utilizaría como revulsivo multidisciplinar la Historia a través de la Arqueología.

Desde estas convicciones y en coherencia a la labor desarrollada durante las últimas décadas dentro y fuera de las aulas universitarias, el Grupo de Investigación en Arqueología de la Universidad de Murcia (en adelante, GIA de la UMU) ha articulado una nueva propuesta didáctica para la enseñanza/aprendizaje de la Arqueología en la Educación Secundaria. Enunciados sus principios básicos (Fernández Díaz et alii, e.p.), pasamos ahora a describir algunas de las líneas de actuación, que han podido ser modeladas ya no solo a partir de un ejercicio de reflexión crítica del que ha emanado un corpus teórico, sino también a su puesta en marcha inicial, mediante una etapa de evaluación diagnóstica en algunos centros educativos destinatarios de esta propuesta de innovación educativa. 


\section{Metodología}

La propuesta del GIA de la UMU parte de una fundamentación teórica y una metodología de trabajo que aúna experiencias académicas y profesionales. Por cuanto se refiere al primer aspecto, cabe reseñar la reformulación de planteamientos docentes sobre la enseñanza-aprendizaje de conocimientos históricos y patrimoniales que supuso el Proyecto de Innovación Educativa "Arqueología del Mediterráneo", incluida en la convocatoria Desarrollo de experiencias de innovación educativa en el contexto de la Convergencia europea-2006 (Ros et alii, 2008), luego desarrollados en la implantación del Grado en Historia y el Máster en Arqueología Aplicada. Gestión Profesional y estrategias de investigación en patrimonio arqueológico de la UMU. Dichos planteamientos fueron incardinados en la aplicación de las nuevas tecnologías al patrimonio arqueológico murciano, colaborando estrechamente con instituciones y centros vinculados a tal reto, tales como la Fundación Integra o el Centro Mediterráneo de innovación digital en patrimonio cultural, DIGITALMED.

Del mismo modo, el diseño de nuestra propuesta de innovación no ha sido ajeno a un enfoque crítico, integral e integrado, en la epistemología educativa, de modo que, con la finalidad de insistir en los avances digitales en el registro, tratamiento, análisis de datos, gestión, divulgación y difusión del patrimonio arqueológico, en tanto herramienta para una más eficaz y enriquecedora enseñanza de las ciencias sociales en secundaria, se ha trabajado bajo los estándares TIC para docentes emanados de la ISTE, atendido a las directrices del Programa Escuela 2.0 del Instituto Nacional de Tecnologías Educativas y Formación de Profesorado (INTEF) del MEC.

De esta forma, subyace en nuestro proyecto el trabajo colaborativo y la generación de conocimiento compartido a través del estímulo a la interactuación entre los agentes de la comunidad educativa (Martínez Sánchez, 2007), contrastando ejemplos cosechados en otros proyectos de innovación educativa aún en disciplinas científicas diversas (Martínez, Corzana y Millán, 2013).

Igualmente, en esta fundamentación teórica y articulación de estrategias metodológicas capaces de asegurar las metas formativas perseguidas, el GIA de la UMU ha asumido igualmente los principios de la didáctica en la Educación Secundaria tanto del denominado "patrimonio integral" (Ballesteros et alii, 2003; Sánchez, 2012) como, de forma específica, del patrimonio arqueológico (Bardavio et alii, 1996; Santacana, 1996; Bardavio et alii, 2003; y García et alii, 2003).

Muy especialmente, para la adopción de una metodología pedagógica que contemple la implementación de la Arqueología en los currículos de las aulas preuniversitarias, se han seguido experiencias como las desarrolladas por el Laboratorio de Arqueología y Enseñanza de la UAB, integrado desde 2002 en el Centro de Estudios sobre el Patrimonio Arqueológico de la Prehistoria (CEPAP) de la mencionada institución universitaria (http://cepap.uab.cat/).

Igualmente, entre los modelos de referencia cabe citar diversas iniciativas promovidas por el Institut Català d'Arqueologia Clàssica (ICAC) en colaboración con otros 
entidades. Se trata de programas que, al igual que la propuesta que es objeto de este trabajo, se enfocan tanto al profesorado como al alumnado de Educación Secundaria. Así, en el primer caso, consideramos una experiencia interesante el curso "Aprenent del passat" organizado por el ICAC y el Institut de Paleoecologia Humana i Evolució Social (IPHES), en tanto, partiendo de premisas teóricas similares a las nuestras, se dirige al profesorado de secundaria no estrictamente del campo de Humanidades, sino genéricamente de Ciencias, Tecnología y Matemáticas.

Respecto a las iniciativas planteadas para el alumnado de secundaria, junto a actividades como Viatje al passat!, organizados por los referidos ICAC e IPHES con el respaldo de la URV, cabe destacar el programa "Bojos per l'Arqueologia!", incluido en la propuesta formativa "Bojos per la ciencia 2013" promovida por la Fundació Catalunya - La Pedrera. Dicha iniciativa, alumbrada en el marco de las fructíferas sinergias entre el ICAC e IPHES, así como el Institut Català de Recerca en Patrimoni Cultural (ICRPC) persigue, de forma semejante a la propuesta de innovación educativa del GIA de la UMU, fomentar la vocación por la Arqueología y la conservación y preservación del patrimonio cultural entre los alumnos de $1^{\circ}$ de Bachiller en Cataluña (http://bojosperlarqueologia.icac.cat/).

Los resultados obtenidos en esta labor de documentación llevada a cabo por el GIA de la UMU se han articulado en una serie de líneas de actuación que, asumiendo que en los esquemas del trabajo colaborativo la geografía del aprendizaje se expande más allá del espacio físico del aula, se vertebran a partir de una metodología pedagógica que combina las teorías piagetianas del aprendizaje constructivista con los principios del aprendizaje significativo definido por Ausubel (Friera, 1995: 79-81).

Finalmente, dichos razonamientos se han contrastado con la experiencia concreta de dos centros educativos de secundaria de la Región de Murcia, cuyos docentes han sido encuestados bajo el modelo de cuestionario descriptivo con fines explicativos.

\section{Directrices didácticas}

La puesta en marcha de la propuesta de innovación educativa del GIA de la UMU contempla como núcleo fundamental, una etapa formativa que versa sobre la aplicación de las TIC en Arqueología, en las modalidades de registro, conservación, revalorización y comunicación de los recursos patrimoniales arqueológicos, a través de la programación de seminarios. No en vano, si bien la comunidad docente concibe el patrimonio como recurso didáctico, también alerta de los problemas de su aplicación efectiva, postulando una formación eficaz (Fernández, 2003; Prats, 2003; González, 2007). En el caso concreto de la Región de Murcia, partimos además de estudios que evidencian la escasa utilización de las potencialidades de los recursos patrimoniales en la práctica docente (Monteagudo et alii, 2009). 
Ya en esta fase formativa e, igualmente, volcando los resultados de la misma, nuestra propuesta prevé el trabajo mediante blogs confeccionados según los estándares de este tipo de bitácoras con fines educativos en entornos de aprendizaje (Prendes, 2007; y Solano y Gutiérrez, 2007). Dichos blogs, centrados en la docencia práctica y siguiendo los parámetros de las estrategias comunicativas en la gestión patrimonial, seguirían una estructura colaborativa y se integrarían de microcontenidos, persiguiendo la interacción de los usuarios, docentes y discentes (Castañeda, 2007). Estas bitácoras versarían sobre los elementos integrantes del patrimonio arqueológico de la Región de Murcia, concibiendo los distintos yacimientos, colecciones y museos como recursos didácticos (Pérez, 1999).

No obstante, de forma coherente al planteamiento y objetivos de nuestra iniciativa ya desglosada con anterioridad (Fernández et alii, e.p.), tras la fundamentación teórica hemos querido partir de una primera etapa de diagnóstico acerca del grado de conocimiento y experiencias de los docentes de enseñanza secundaria en Arqueología y TIC, en cuyos resultados centraremos nuestro discurso. Se trataba de contrastar nuestras hipótesis con el día a día de las aulas preuniversitarias, intentando que la constatación de rutinas, comportamientos y actitudes permitiera calibrar los puntos débiles y/o fuertes de la propuesta del GIA de la UMU.

Dicho diagnóstico ha consistido en un modelo de encuesta descriptiva. Su objetivo era obtener una visión general sobre cómo utilizan los docentes en Secundaria las herramientas y/o recursos existentes en red como sistema de aprendizaje sobre el patrimonio en sus prácticas educativas, dentro y fuera de los centros, la valoración que profesores y alumnos hacen en este momento de tales experiencias, y las expectativas que una profundización en tales aspectos genera, en tanto proceso de innovación y adaptación del sistema educativo a las nuevas necesidades de una sociedad global $\mathrm{y}$ en continuo cambio.

El diseño del cuestionario, que integra 17 ítems, se divide en dos bloques fundamentales. El primero agrupa la información relativa al perfil de los docentes y de los centros en que desempeñan su labor profesional. Aquí, los ítems relacionados con situación laboral, experiencia profesional o franja de edad de los encuestados, se han planteado bajo la modalidad de preguntas cerradas o estructuradas, ya dicotómicas ( 2 opciones) ya en abanico (3 o más opciones).

El segundo bloque aglutina los ítems referentes a la disponibilidad de las herramientas/recursos, organización de los centros para su uso, cualificación y formación de los docentes de los mismos, la presencia de éstos en las prácticas pedagógicas o la valoración que hacen de ellos los profesionales. En este caso se combinan preguntas cerradas, abiertas o no estructuradas, de profundización y parcialmente estructuradas.

En cuanto a la recogida de la información, hemos planteado un muestreo dirigido o de juicio, así, durante el curso académico 2013/2014, se procedió a seleccionar dos institutos de la Región de Murcia ubicados en entornos de enseñanza aprendizaje diversos: uno de ellos emplazado en una ciudad donde la presencia del patrimonio arqueológico es abrumadora, Cartagena (IES San Isidoro, Los Dolores), y otro en 
el que tal realidad no está tan presente en la vida cotidiana, Molina de Segura (IES Cañada de la Eras).

Se planteó un tamaño muestral de 130 docentes, de los cuales, finalmente, sólo participaron un total de 73 docentes, 29 de Cartagena y 44 de Molina de Segura. Uno de los ítems desglosa tal población, de modo que 44 docentes pertenecen a Ciencias Sociales, 18 a Ciencias Naturales y 11 a otras en las que se incluye Música y Religión (fig. 1A).

Aunque somos conscientes de que el tamaño de la muestra llama a la prudencia a la hora de extrapolar sus resultados, creemos que se ha conseguido abarcar un sector diverso de la comunidad docente de enseñanza secundaria en la Región de Murcia.

De hecho, redunda en tal apreciación no ya solo el campo disciplinar al que pertenecen los profesores o el contexto de "cotidianeidad" patrimonial en el que trabajan, sino su misma franja de edad o experiencia docente. Así, en referencia al primer aspecto, el grupo más elevado es el que se encuentra entre 36 y 55 años, siendo el más numeroso el de ciencias sociales, y acercándose el de ciencias naturales en el intervalo de 46 a 55 años. En consonancia, 12 docentes cuentan con una experiencia menor o igual a 10 años, 33 al intervalo entre 11 y 20 años, 18 a la banda entre 21 y 30, o 9 entre 31 y 40 años (figs. 1B y 1C).

En cuanto a la frecuencia con que utilizan las herramientas TIC, de los 73 participantes, prácticamente todos (70) hacen uso de estos recursos fuera del aula con una intensidad que varía entre mucho y suficiente. En tal segmento, destacan los docentes cuyo itinerario es el de la Ciencias Sociales.

Fig. 1.- Gráficos de resultados sobre las cuestiones siguientes: A) ¿En qué ámbito se desarrolla su actividad docente? B) ¿Grupo de edad en el que se encuentra? C) Cuantifica tus años de experiencia laboral en las aulas de E. Secundaria D) ¿Con qué finalidad utilizas las TIC?

A)
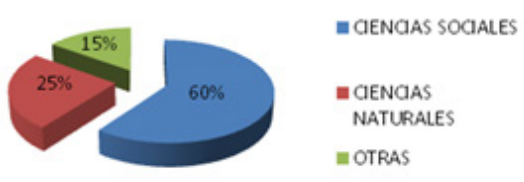

=OTRAS

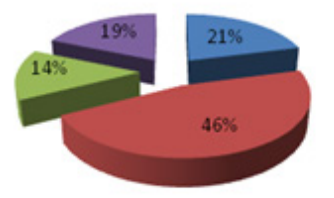

I $11-20$

$=21-30$

$=31-40$
B)

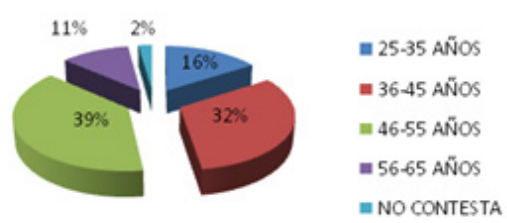

D)

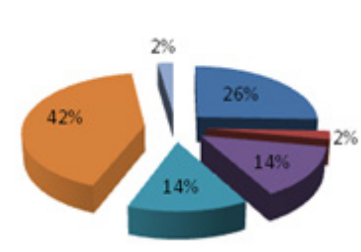


Sobre la disponibilidad de recursos y/o herramientas en el aprendizaje y comunicación del patrimonio, queríamos rastrear la disponibilidad en los centros de enseñanza de recursos o herramientas TIC para el aprendizaje y comunicación del patrimonio, para qué los utilizan (preparación de clases, gestión de programas, memorias...) o, en su defecto, el por qué no (Fig. 3B). En este caso, una mayoría de encuestados (71\%) considera adecuada la infraestructura de sus centros, si bien tampoco cree que sea la idónea. En la misma línea, un porcentaje similar (70\%) señala un uso cotidiano de las TIC en su práctica docente, focalizado sobre todo en la preparación de las clases, pero también en la gestión administrativa (fig. 1D). De forma coherente a tales respuestas, todos los encuestados ve beneficios tangibles en la aplicación de las TIC a la labor docente, cifrando éstas como muy positivas (75\%) o al menos, suficientes (25\%) (Fig. 3A). De hecho, a la hora de concretar qué herramientas utilizan, cuestión que se planteó abierta, nos ha sorprendido gratamente su diversidad (plataformas educativas, entornos virtuales de aprendizaje, redes sociales, software educativos y otros), añadiendo además, que el empleo de estas aplicaciones y no otras responde no tanto a carencia de infraestructuras en los centros, como sí a la falta de tiempo (20\%) o la convicción de que sus resultados no son plenamente fructíferos en el proceso de enseñanza/aprendizaje (38\%).

Fig. - 2. Gráficos de resultados sobre las cuestiones siguientes: A) ¿Si no utiliza las TIC ve beneficios en su uso y cree que facilitarían la tarea docente? B) ¿Cuál es el motivo por el que no utiliza el resto de herramientas TIC?

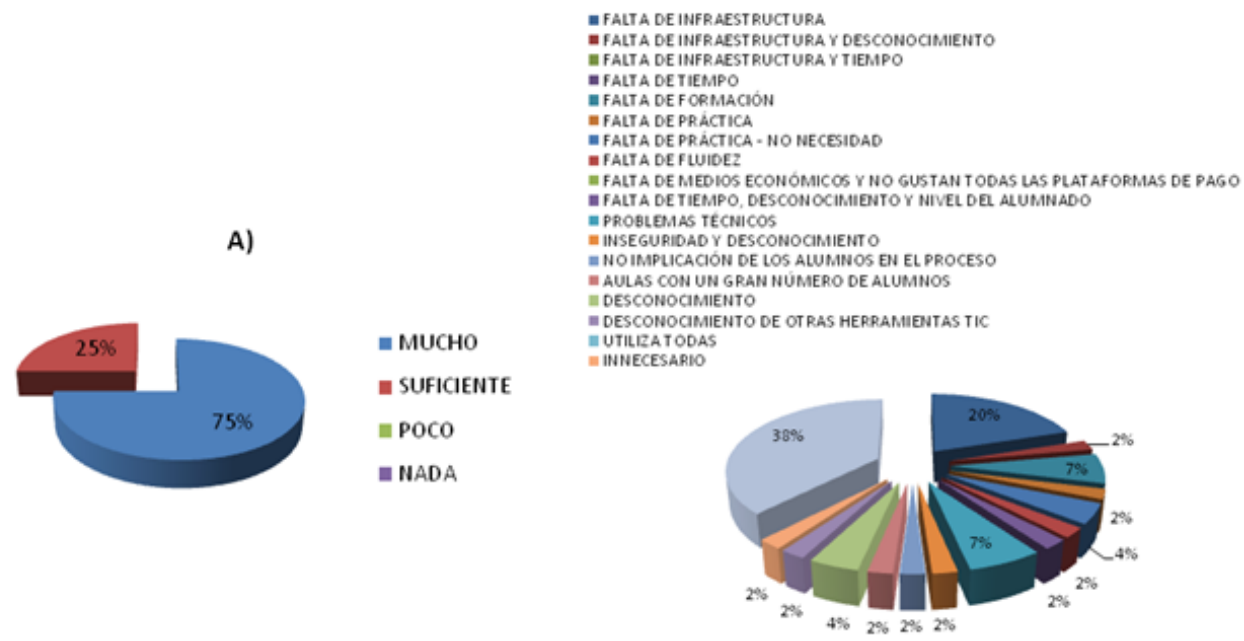

En el campo de cualificación y formación de los docentes en esas herramientas/ recursos para el aprendizaje y comunicación del patrimonio, una holgada mayoría (59\%) considera insuficiente el apoyo que presta la administración educativa en el 
manejo de las TIC aplicadas a la enseñanza/aprendizaje, abogando consecuentemente de forma abrumadora (93\%) por programas formativos más eficaces.

Finalmente, también hemos pedido a los docentes su valoración explícita acerca de la propuesta del GIA de la UMU, consultando acerca de si ven las potencialidades de la disciplina arqueológica como ciencia integradora de la Historia con otras disciplinas, con los implícitos beneficios implícitos en la mejora cualitativa de las estrategias de enseñanza/aprendizaje que estimularía su implementación en el currículo de la enseñanza preuniversitaria. De nuevo nos ha sorprendido gratamente la respuesta positiva, pues, una mayoría respalda tales iniciativas, con un alto grado de satisfacción (50\%) o al menos suficiente (34\%), considerándola altamente beneficiosa (39\%) o al menos moderadamente provechosa (34\%) para el alumnado (figs. 3A-C).

De hecho, estimula que junto a ese recibimiento, también mayoritariamente se encuentre interesado en conocer más acerca de estas cuestiones, que creen poco (34\%) o nada (25\%) trabajadas en sus entornos educativos, siendo significativo que la mayor satisfacción en casi todos los ítems, se registre entre los docentes de Ciencias Sociales.

Fig. 3.- Gráficos de resultados sobre las cuestiones siguientes: A) ¿Cree necesarias nuevas sesiones formativas sobre las ventajas de las TIC en el proceso de enseñanza/aprendizaje?

B) ¿Cree factible desarrollar el proyecto Arqueología y TIC en Enseñanza Secundaria en el marco en el marco de las asignaturas que imparte? C) ¿Cree que, dentro de los parámetros de enseñanza/aprendizaje colaborativos, desarrollar contenidos sobre esta Arqueología científica, podría ayudar a la consecución de las competencias contempladas en las asignaturas que imparte?

A)

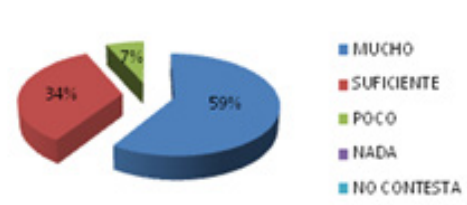

B)

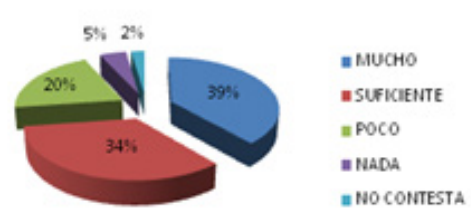

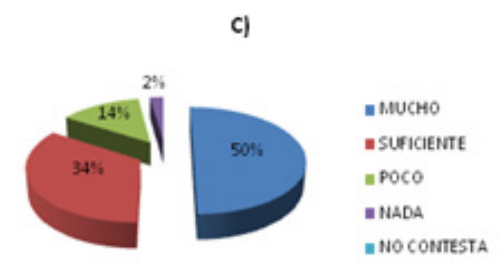




\section{Conclusiones}

Los resultados de esta etapa diagnóstica, la constatación de necesidades formativas y las expectativas generadas en el campo de la enseñanza/aprendizaje de la Arqueología a través de las TIC en las aulas de secundaria, alientan a continuar con este proyecto de innovación educativa.

No en vano, si ya la comunidad docente es consciente de las ventajas de implementar la didáctica de tal disciplina científica en la enseñanza primaria (Rivero, 2008), su idoneidad se hace más evidente en la siguiente etapa educativa, donde la propuesta del GIA de la UMU, insistiendo en el carácter interdisciplinar de la Arqueología, podría fomentar un aprendizaje más innovador, transversal e integrador. De hecho, ya en sí la exclusiva enseñanza / aprendizaje del método arqueológico en este nivel se perfila como una de las experiencias potencialmente más fructíferas para desarrollar el conjunto de procedimientos, habilidades y destrezas cognitivas que favorecen la consecución de un pensamiento explicativo, interpretativo y crítico entre los discentes (Bardavio et alii, 2003). Si a ello unimos una visión más ambiciosa que incardine la apreciación del patrimonio arqueológico en paisajes vividos, impulsaríamos no solo el desarrollo de valores y actitudes relacionadas con la valoración y conservación del patrimonio histórico-arqueológico, sino la misma implementación de tareas procedimentales básicas (identificar, describir, comparar, clasificar, interpretar) contempladas en sus curricula.

Evidentemente, dichos objetivos, con el horizonte final de conseguir resultados educativos de calidad, se abordarían en el marco del desarrollo y estímulo de la competencia digital, propiciando el manejo de medios y herramientas de la Educación 2.0.

Así, bajo las premisas de las TAC, la propuesta de innovación educativa del GIA de la UMU persigue no tanto asegurar el dominio de determinadas herramientas informáticas, como sí incidir en la metodología y usos didácticos de las nuevas tecnologías en materia arqueológica (Barba y Capella, 2010). Insistimos, abordando las dimensiones tecnológico-instrumental, comunicacional, colaborativa, cognitiva, creativa o axiológica, concebimos nuestra propuesta formativa en Arqueología, en sus vertientes actitudinal, procedimental o instruccional, capacitando, respectivamente, en el manejo de técnicas colaborativas, interactivas y expositivas. Todos asumimos que algunas de las dificultades en el aprendizaje de los discentes residen no tanto en la falta de capacidad como sí en la necesidad de articular estrategias educativas eficaces que, entre otros aspectos, dada la condición de "nativos digitales" de nuestros alumnos, se adecúen a tales realidades (Seva et alii, 2010).

Igualmente, como ya explicitamos (Fernández et alii, e.p.), nuestro proyecto pretende focalizar tales metas en el patrimonio arqueológico del Sureste hispano, concebido por el GIA de la UMU como un laboratorio "vivencial", en tanto, el mismo entorno local, lejos de visiones simplistas o particularistas, se puede y debe convertir en el escenario para la búsqueda, registro y análisis de testimonios específicos, tangi- 
bles y, a fin de cuentas, físicamente accesibles, que plasmen los contenidos factuales y estimulen el aprendizaje procedimental y el desarrollo de habilidades cognitivas (Bardavio et alii, 2003: 490-491).

En suma, creemos que la puesta en marcha de la propuesta didáctica Arqueología y TIC del GIA de la UMU, promovería una experiencia educativa constructivista en el ámbito académico preuniversitario, favoreciendo el progresivo alejamiento de la tradicional heteronomía del discente, con el desarrollo de un pensamiento crítico y creativo, bases del conocimiento participativo y autónomo (Santisteban, González y Pagès, 2010: 119).

A través de espacios y herramientas digitales y dentro de los nuevos esquemas de trabajo colaborativo, se pretende insistir en los principios de investigar, innovar, gestionar y compartir el conocimiento en el campo del patrimonio arqueológico, facilitando la formación de orientadores capaces de estimular el aprendizaje compartido en Arqueología, como disciplina para una más enriquecedora enseñanza de las ciencias sociales en las aulas de secundaria.

\section{Bibliografía}

BARBA, C.; CAPELLA, S. (coords.). 2010. Ordenadores en las aulas. La clave es la metodología. Barcelona.

BARDAVIO, A.; GATELL, C.; GONZÁLEZ, P. (2003). "El patrimonio arqueológico local como tema de investigación en la Educación Secundaria post-obligatoria". En: BALleSTEROS, E. (et al.) (coords.). El Patrimonio y la Didáctica de las Ciencias Sociales. Cuenca, p.489-498.

BARDAVIO, A.; GATELL, C.; MOLINERO, F. (1996). "Recerca I divulgació arqueològica. Un camí per al desenvolupament d'estratègies interdisciplinars a l'ESO". En: Treballs d'Arqueologia, 4, p.50-76.

BARDAVIO, A.; GONZÁLEZ MARCÉN, P. (2003). Objetos en el tiempo. Las fuentes materiales en la enseñanza de las ciencias sociales. Barcelona.

(2008). "La Arqueología y la Prehistoria como eje en un proyecto integrado de comprensión del territorio. El ejemplo del campo de aprendizaje de La Nogueruela". En: ÁVILA, R.M ${ }^{\mathrm{a}}$; CRUZ, A.; DIEZ, M ${ }^{\mathrm{a}}$ C. (eds.). Didáctica de las Ciencias Sociales, Currículo Escolar y Formación del Profesorado. La didáctica de las Ciencias Sociales en los nuevos planes de estudio. Jaén, p.473-484.

CASTAÑEDA, L. (2007). "Software social para la escuela 2.0: más allá de los Blogs y las Wikis". En: Inclusión Digital en la Educación Superior: Desafios y oportunidades en la sociedad de la Información. X Congreso Internacional EDUTEC 2007. Edición electrónica. Buenos Aires, Universidad Tecnológica Nacional.

FERNÁNDEZ DÍAZ, A.; VIZCAÍNO SÁNCHEZ, J.; ROS SALA, Ma M.; RAMALLO ASENSIO, S.F. (e.p.). "Propuesta didáctica para la enseñanza/aprendizaje de la Arqueología en la Educación Secundaria. Experiencias del Grupo de Investigación en Arqueología de la Universidad de Murcia (España)" 
FERNÁNDEZ SALINAS, V. (2003). "Escenarios para el aprendizaje del patrimonio". En: BALLESTEROS, E. (et al.) (coords.). El Patrimonio y la Didáctica de las Ciencias Sociales. Cuenca, p.281-289.

FRIERA SUÁREZ, F. (1995). Didáctica de las Ciencias Sociales: Geografía e Historia. Madrid.

GONZÁLEZ MONFORT, N. (2007). "El valor educativo y el uso didáctico del patrimonio cultural". En: Educación primaria. Orientaciones y recursos (6-12 años). Barcelona, p.207-262.

MARTÍNEZ, R.; CORZANA, F.; MILLÁN, J. (2013). "Experimentando con las redes sociales en la enseñanza universitaria en ciencias". En: Revista Eureka sobre Enseñanza y Divulgación de las Ciencias, vol.10, n³, p.394-405.

MARTÍNEZ SÁNCHEZ, F. (2007). "La integración escolar de las nuevas tecnologías". En: CABERO, J. (coord). Nuevas Tecnologías aplicadas a la Educación. Madrid, pp. 22-40.

MONTEAGUDO FERNÁNDEZ, J.P.; MIRALLES MARTÍNEZ, P.; MARTÍNEZ VALCÁRCEL, N. (2009). "La formación ciudadana a través de las salidas didácticas para visitar el patrimonio cultural. Un estudio empírico en la Región de Murcia". En: ÁVILA, R.M.; BORGHI, B.; MATTOZZI, I. (coords.). L'educazione alla cittadinanza europea e la formazione degli insegnanti. Bologna, p.405412.

PÉREZ JUEZ, A. (1999). "El yacimiento como recurso didáctico: acondicionamiento, restauración, reconstrucción y otras ideas". En: Apuntes de Arqueología, $\mathrm{n}^{\circ} 7, \mathrm{p} .19-22$.

PRATS, J. (2003). "Líneas de investigación en didáctica de las ciencias sociales". En: Historia \& Ensino. Revista do Laboratorio de Ensino de Historia, nº 9, p.1-25.

PRENDES, $M^{a}$ P. (2007). "Internet aplicado a la educación: estrategias didácticas y metodologías". En: CABERO, J. (coord.). Nuevas tecnologías aplicadas a la educación. Madrid, p. 205-222.

RIVERO GRACIA, M ${ }^{\mathrm{a}}$ P. (2008). "Propuesta para una integración del patrimonio arqueológico en el nuevo currículo de educación primaria". En: ÁVILA, R.M ${ }^{\text {a }}$; CRUZ, A.; DIEZ, M ${ }^{\text {a }}$ C. (eds.). Didáctica de las Ciencias Sociales, Currículo Escolar y Formación del Profesorado. La didáctica de las Ciencias Sociales en los nuevos planes de estudio. Jaén, p.391-400.

ROS SALA, $\mathrm{M}^{\mathrm{a}} \mathrm{M}$. et alii (2008). "Proyecto de innovación educativa "Arqueología del Mediterráneo". En: ARNAIZ, P.; HERNÁNDEZ, L.; GARCÍA, Mª P. (coords.): Experiencias de innovación educativa en la Universidad de Murcia (2007). Murcia, p.339-364.

SÁNCHEZ HIDALGO, C.I. (2012). El patrimonio como recurso didáctico en la Educación Secundaria y Bachillerato. Murcia.

SANTACANA, J. (1996). "La interrelació entre docència i investigación en arqueología". En: Treballs d'Arqueologia, 6, p.8-38.

SOLANO, I.M. y GUTIERREZ, I. (2007). "Herramientas para la colaboración en la enseñanza superior: blogs y wikis". En: PRENDES, M.P. Herramientas telemáticas para la enseñanza universitaria en el marco del Espacio Europeo de Educa- 
ción Superior. Grupo de Investigación de Tecnología Educativa. Universidad de Murcia.

SANTISTEBAN, A.; GONZÁLEZ, N.; PAGÈS, J. (2010). "Una investigación sobre la formación del pensamiento histórico". En: ÁVILA RUIZ, R. M ${ }^{\mathrm{a}}$; RIVERO GRACIA, MaP.; DOMÍNGUEZ SANZ, P.L. (coords.). Metodología de Investigación en Didáctica de las Ciencias Sociales. Zaragoza, p.115-128.

SEVA CAÑIZARES, F.; SORIANO LÓPEZ, M.C.; VERA MUÑOZ, M.I. (2010). "La práctica docente en las Ciencias Sociales: un análisis cuantitativo". En: ÁVILA RUIZ, R. M ${ }^{\mathrm{a}}$;; RIVERO GRACIA, Mª.; DOMÍNGUEZ SANZ, P.L. (coords.). Metodología de Investigación en Didáctica de las Ciencias Sociales. Zaragoza, p.175-183.

\section{Los autores}

Alicia Fernández Díaz es Dra. en Arqueología e Historia Antigua. Trabaja como Prfra. Titular del Área de Arqueología de la UMU, ejerciendo su actividad docente en el Grado de Historia así como en varios Doctorados y Másteres de la UMU: Máster de Arqueología Aplicada. Gestión profesional y estrategias de investigación en patrimonio arqueológico, Máster de Educación y Museos. Patrimonio, identidad y mediación" (modalidad on-line)) y Máster de Historia Naval. Ha sido Secretaria de Departamento, de la Comisión de Relaciones Internacionales de Letras, tutora y coordinadora Erasmus, y actualmente es Secretaria de DIGITMED. Relativos a la temática sobre la que versa este trabajo, ha colaborado en 2 Proyectos de Innovación docente: "Coordinación de contenidos fundamentales de la Titulación de Historia dependientes del Departamento" y "Acción especial (modalidad B). Arqueología del Mediterráneo". En los tres últimos años, ha participado en el Máster de Educación y Museos de la Universidad de Murcia, en el que prima la utilización constante de las herramientas y recursos TIC (web 2.0, foros, chats, wikis...), así como en la asignatura de TIC para la Historia.

Jaime Vizcaíno Sánchez es Dr. en Arqueología. Trabaja como Prof. Asociado en el Área de Arqueología de la UMU, ejerciendo su actividad docente en el marco del Grado en Historia y Grado en Historia del Arte, así como en el Máster de Arqueología Aplicada. Gestión profesional y estrategias de investigación en patrimonio arqueológico. Ha colaborado en el Proyecto de Innovación docente: "Acción especial (modalidad B). Arqueología del Mediterráneo". Forma parte del equipo docente de la asignatura TIC para la Historia. Junto a sus líneas de investigación en Arqueología de la Antigüedad Tardía, aspectos materiales de época protobizantina y Cristianismo primitivo, destaca su colaboración con instituciones museísticas en el diseño y producción de diferentes iniciativas y recursos didácticos.

$M^{a}$. Milagrosa Ros Sala es Dra. en Arqueología e Historia Antigua. Trabaja como Prfra. Titular de Prehistoria de la UMU, ejerciendo su actividad docente en Prehistoria y Arqueología en Licenciatura y Grado de Historia, Doctorados y Masters 
("Arqueología Aplicada. Gestión profesional y estrategias de investigación en patrimonio arqueológico", "Educación y Museos. Patrimonio, identidad y mediación" (modalidad on-line)). Coordinadora del Laboratorio de Arqueología de la UMU, ha sido Investigadora Coordinadora del Proyecto de Innovación Educativa "Arqueología del Mediterráneo" (UMU) (Desarrollo de Experiencias de Innovación Educativa en el contexto de la Convergencia Europea (UMU), 2006/2007) e Investigadora en Proyectos Internacionales: "Estudios de postgrado en educación y museos. Patrimonio, identidad y mediación cultural" 2010/2011 y "Acción preparatoria entre la Universidad de Murcia y la Universidad Cadi-Ayyad (Marrakesch) para el desarrollo de una metodología integrada para la formación de expertos en gestión e interpretación del patrimonio" 2010/2011.

Sebastián F. Ramallo Asensio es Dr. en Arqueología e Historia Antigua. Trabaja como Catedrático de Arqueología de la UMU, ejerciendo su actividad docente en Arqueología en Licenciatura y Grado de Historia, Doctorados y Masters: Máster en Arqueología Aplicada. Gestión profesional y estrategias de investigación en patrimonio arqueológico" y Doctorado de Arqueología, Paisajes y territorios históricos en el Mediterráneo y Sureste de la Península Ibérica, de los cuales es coordinador, así como en el Máster de Educación y Museos. Patrimonio, identidad y mediación (modalidad on-line)) o en el Máster de Historia Naval de la UMU. Investigador en Proyecto de Innovación Educativa "Arqueología del Mediterráneo" (UMU) (conv. Desarrollo de Experiencias de Innovación Educativa en el contexto de la Convergencia Europea (UMU), 2006/2007). Investigador en Proyectos Internacionales: "Estudios de postgrado en educación y museos. Patrimonio, identidad y mediación cultural" 2010/2011 y "Acción preparatoria entre la Universidad de Murcia y la Universidad Cadi-Ayyad ( Marrakesch) para el desarrollo de una metodología integrada para la formación de expertos en gestión e interpretación del patrimonio" 2010/2011. 